\title{
Study for the Development of A System of Measurement of the Dynamic Characteristics of Trans-Femoral Prostheses: Measurements of Differing Knee Joint Characteristics
}

\author{
Takeshi Tsuruga, Hiroyuki Iino, Akihiko Takashima, Kanou Yoshida, \\ Tomohiro Nomura, Hiroyuki Matsubara
}

\begin{abstract}
Test subject participation has generally been essential in assessing the performance of trans-femoral prosthetic components, but it inherently poses the problem of test subject gait fatigue hindering reproducibility, limits reiteration number, and introduces non-objective aspects. We are conducting a series of studies to develop a system for trans-femoral prostheses evaluation to assess knee joints with previously unattainable objectivity. In the present study, we apply this system in its present form to measure the simulated gait actions of trans-femoral prostheses containing different knee joints, one with a bouncing mechanism and the other with a weight stabilizing mechanism. The results show that the system can provide measurements of the characteristics of the differing knee joints in respect to ground reaction force and joint angles.
\end{abstract}

Index Terms - trans-femoral prostheses, knee joint unit, simulated gait, ground reaction force

\section{INTRODUCTION}

Trans-femoral prostheses are distinguished from other prostheses particularly by their incorporation of a prosthetic knee joint in place of the natural knee joint, and the related difficulty of gaining gait ability that requires extensive training, practice, and familiarization with the specific characteristics of the knee joint. Recent years, moreover, have seen the development of knee joints with wide-ranging functions, and gait analysis by test subjects wearing the lower-extremity prostheses in clinical on-site assessments of their characteristics has become increasingly important. This can provide important information on the feel of use of the lower-extremity prostheses and other aspects that can be obtained only with a test subject who is actually walking, but

Takeshi Tsuruga, Department of Prosthetics and Orthotics, Faculty of Health Sciences, Hokkaido University of Science, Sapporo, Hokkaido, Japan

Hiroyuki Iino, C.P.O. Sales Department, Imasen Engineering Corporation, Kakamigahara, Gihu, Japan

Akihiko Takashima, Department of Mechanical Engineering, Faculty of Engineering, Hokkaido University of Science, Sapporo, Hokkaido, Japan

Kanou Yoshida, Department of Mechanical Engineering, Faculty of Engineering, Hokkaido University of Science, Sapporo, Hokkaido, Japan

Tomohiro Nomura, Department of Prosthetics and Orthotics, Faculty of Health Sciences, Hokkaido University of Science, Sapporo, Hokkaido, Japan

Hiroyuki Matsubara, Department of Prosthetics and Orthotics, Faculty of Health Sciences, Hokkaido University of Science, Sapporo, Hokkaido, Japan it also involves fatigue-related limitations on reproducibility, limited reiteration number, and other unavoidable problems, and it cannot readily be regarded as objective.

In this light, we are engaged in a series of studies to develop a system for the evaluation of trans-femoral prostheses with knee joints that can in particular determine the dynamic characteristics in a continuous gait mode. The system is directed toward safely and objectively determining the gait characteristics of trans-femoral prostheses incorporating a knee joint and foot under a range of gait conditions (e.g., cadence, step length, hip-joint flexion-extension angle). The use of the present system and its effectiveness in measurement of the kinetic characteristics of trans-femoral prostheses with different knee joints were examined.

\section{KNEE JOINTS}

\section{A. Role And Types}

The knee plays important roles in support of a person's weight, impact absorption, leg swing, and other gait actions. A prosthetic knee joint serves as a substitute for a natural knee joint lost in an accident, illness, or other circumstance. It is obviously desirable for a prosthesis to enable performance of motions and functions matching those of able-bodied individuals, but despite the dramatic progress in recent years, prosthetic knee joints must still be selected for functions in accordance with the residual functions of the user. Knee-joint control varies widely between the stance and swing phases. Specific features in further detail include the following.

1) Stance phase control

- Immobilized knee joint

Knee joint automatically immobilized when fully extended in stance.

- Weight stabilizing knee joint

Frictional force generated and brake applied on knee axis from above and below by application of body weight.

- Bouncing knee joint

Mechanism that provides light flexion of the knee joint seen after initial contact in able-bodied individuals 


\section{Study for the Development of A System of Measurement of the Dynamic Characteristics of Trans-Femoral Prostheses: Measurements of Differing Knee Joint Characteristics}

with reduction of gait energy consumption as an objective.

- Yielding knee joint

Mechanism that slowly flexes the knee joint by the resistance of a hydraulic cylinder when load is applied to the trans-femoral prostheses.

\section{2) Swing phase control}

- Immobilized knee joint

Mechanism that immobilizes the knee joint in stance and gait

- Spring action

Mechanism that uses a spring reaction force for flexion resistance in knee joint flexion and extension assist.

- Mechanical friction unit

Mechanism that applies friction by pinching the knee axis to induce slow flexion and extension.

- Fluid control unit

Mechanism that uses pneumatic and hydraulic pressure to generate resistance in accordance with flexion speed.

These various functions of the knee joint occur in combination with the foot to obtain the diverse characteristics of trans-femoral prostheses.

\section{B. Method of Trans-Femoral Prosthetic Assessment}

The use of a flexible goniometer by Sekikawa et al. for measurement and assessment of the function of stance phase control of various knee joints is representative of the current state of objective assessment of trans-femoral prostheses containing a knee joint, and has elucidated the functions required for knee joints of lower-extremity prostheses in each phase of the gait cycle with a particular focus on the stability of light flexion in the stance phase in the bouncing mechanism of the knee joint [1]. Nosaka et al. have also assessed knee joints for individual function and reported the results of gait analysis for gait on a flat surface [2]. In all of the studies, the gait actions have been performed by amputees or able-bodied individuals, and no experiments have been performed on test rigs.

Yoshida et al., in contrast, have developed a gait function tester focused on the foot alone to objectively assess the performance of prosthesis feet, applied pressure on the foot much as occurs in actual gait, and performed a study that presents the path of the force contact point in a foot coordinate system [3]. As shown by this development, objective assessment of lower-extremity prostheses by machines is currently in progress, but there has been no study on the performance of trans-femoral prostheses assessment on a test rig.

\section{OVERVIEW OF THE PRESENT SYSTEM}

Natural human gait involves repetition of a cycle of actions in movement of parts extending from initial contact on one side to the next initial contact on that side. The lower-extremity movements include hip-joint and knee-joint flexion and extension together with ankle-joint plantar flexion and dorsiflexion, and movements by each component [4]. In trans-femoral prosthetic gait, in contrast, movement from the knee joint to the foot proceeds passively. In the present system, the movement of the femoral component is therefore regarded as the first movement and the object of control by the system. In both able-bodied individuals and femoral amputees, moreover, visible vertical movement of the center of mass also occurs while gait and was taken as a control parameter. A test performed on the above basis indicated the overview shown in Fig. 1 for the gait action with the present system. The system motors were interlocked based on movement data obtained with actual gait action, to impart the simulated gait action.

Fig. 2 shows the appearance of the system as constructed for the present study. For measurements with the trans-femoral prostheses, it was attached to and used with the simulated femur corresponding to the human femoral region. Three AC servomotors (one each for simulated femoral swing, simulated femoral vertical movement, and force plate reciprocation) were incorporated into the present system and interlocked to obtain the simulated gait action [5].

The control algorithm for each motor engages position control with hip-joint movement as the starting point. The hip-joint flexion-extension angle that is to be attained and the angular speed of the hip joint that will contribute to the cadence are first set. On this basis, the time of action in one gait cycle and the vertical speed of the center of mass in conjunction with the set vertical stroke of the center of mass are calculated, and the motor is operated. Based on the above action time and hip-joint flexion-extension angle together with the prosthesis length, the amplitude and speed for the forward-rearward movement direction of the force plate are calculated and controlled. The programming of servomotor interlocking in this measurement system was performed on Microsoft Visual Basic 2008 because of its ease of construction

\section{COMPARISON OF KNEE JOINTS}

\section{A. Experimental Objective}

The experiment was performed to verify that the characteristics of each knee joint could be measured in the simulated gait, with the knowledge that interchanging knee joints strongly affects the stance and swing phases of gait.

\section{B. Experimental Conditions}

1) Materials

The 3R60 (Otto Bock, Duderstadt, Germany) knee joint

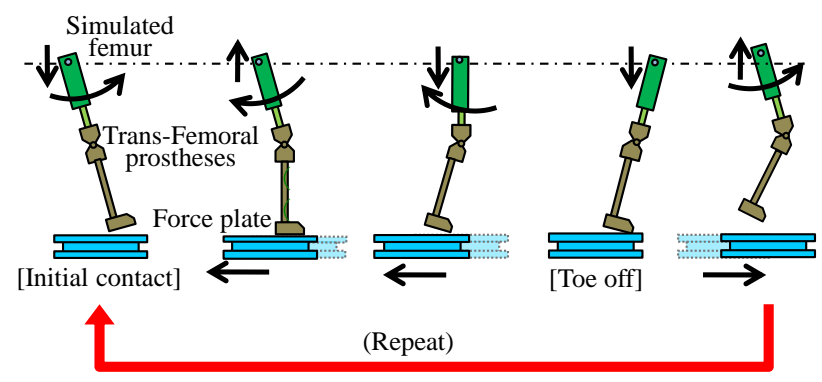

Fig. 1. Overview of present system 
(Fig. 3, left) was tested first, since it facilitates control of flexion-extension resistance, is stable in initial contact while gait, and incorporates a bouncing mechanism [6]. Next to be tested was the BASS (Imasen Electric Industrial Co., Ltd., Aichi, Japan) knee joint (Fig. 3, center) [7], which differs structurally from the $3 \mathrm{R} 60$ in its single-axis rotational center configuration and permits relatively easy adjustment of its stance and swing phases. Flexion-extension control adjustment was performed for each knee joint in accordance with the cadence in this experiment. With consideration for equipment safety in this initial experiment, the energy-accumulating J-Foot (Imasen Electric Industrial Co., Ltd., Aichi, Japan) (Fig. 3, right) [7], which can readily deform and absorb energy under excessive force, was used.

\section{2) Control-parameter setting entry}

In light of the structural limitations of the present system, the prosthesis size was that of female adults. More specifically, the assumed dimensions were based on the mean height of adult women $(158 \mathrm{~cm})$, with leg length of $80.5 \mathrm{~cm}$ and foot length of $22 \mathrm{~cm}$. The settings for hip-joint angles in gait were chosen with consideration of the status of this as the initial experiment, as $15^{\circ}$ in flexion and $10^{\circ}$ in extension and thus less than the mean values for everyday gait of 20 to $30^{\circ}$ in flexion and 10 to $15^{\circ}$ in extension. The gait speed was set to $60 \mathrm{steps} / \mathrm{min}$ and thus somewhat lower

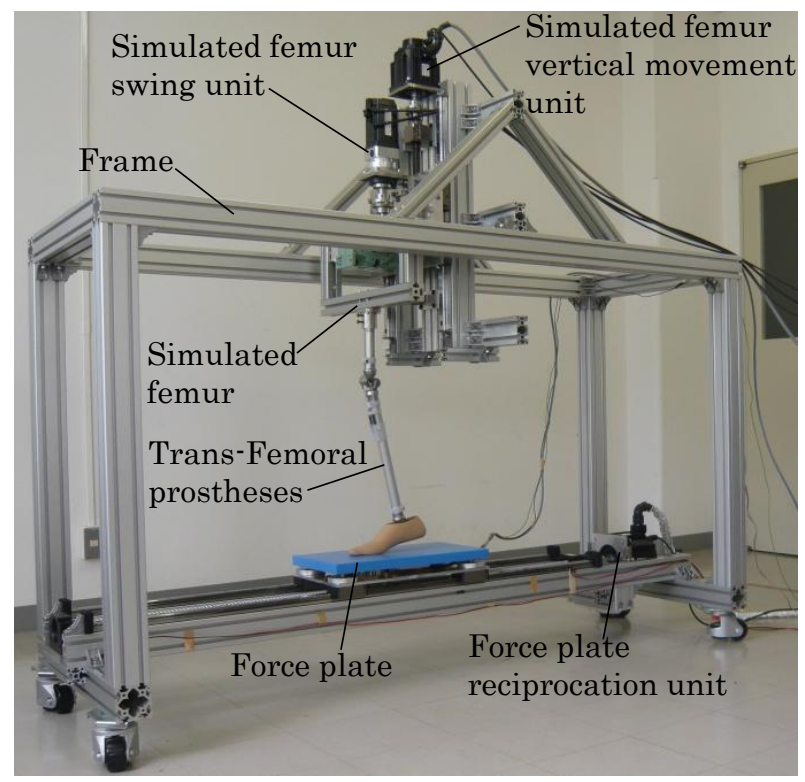

Fig. 2. Appearance of present system
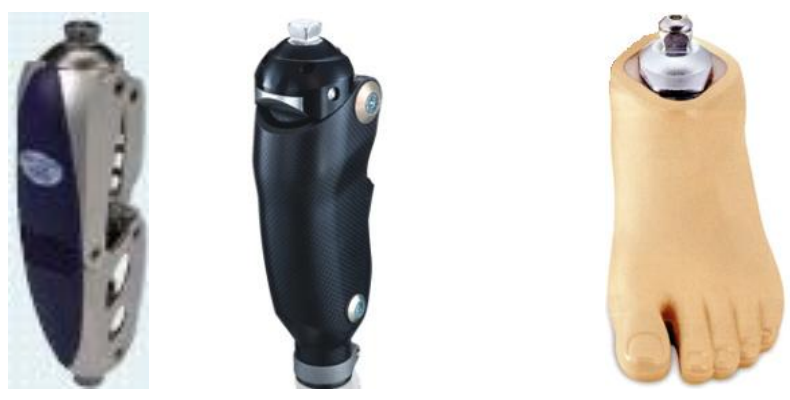

Fig. 3. Knee joints and foot used (Left: 3R60; Center: BASS; Right: J-Foot) than the mean gait speed of 90 to 120 steps/min.

The motors were set in accordance with the human gait action based on these settings [8]. Specific settings included an angular speed of $28 \%$ for the simulated femoral swing motor in extension and, with reference to human gait action, $72.6 \%$ in flexion, and thus substantially faster than in the swing phase to maintain foot clearance from the force plate in the swing phase of knee-joint flexion and toe off.

The motor for simulated femoral vertical movement was set for a stroke value of $1 \mathrm{~cm}$, with the position to reach the highest point at mid stance, mid swing, and hip joint maximum flexion, and the lowest point at hip joint maximum extension.

The motor for ground reaction force reciprocation initiated rearward movement after initial contact, with the speed calculated from the hip joint angular speed and the prosthesis length. The hip joint then undergoes maximum extension and transitions to flexion and then, after confirmation of toe off, moves independently (at 5 times the stance-phase speed) to match the next initial contact.

A flexible goniometer (M180, DKH Co.,Ltd., Tokyo, Japan) was attached to the knee joint, and the knee-joint flexion-extension angle during the simulated gait action was measured in correspondence with the force plate value at a sampling frequency of $100 \mathrm{~Hz}$ via an incorporated A/D converter (AIO-163202F-PE, Contec Co., Ltd., Osaka, Japan).

\section{EXPERIMENTAL RESULTS AND DISCUSSION}

\section{A. Fine Adjustment of Control Parameters}

When common parameters were set and the gait action was performed, with the BASS it was difficult to maintain the angle of maximum extension in the latter stage of the swing phase, and in maximum extension of the knee, terminal impact occurred with the knee joint flexed under rebound, affecting the initial contact and preventing establishment of the simulated gait action. To synchronize the initial contact and maximum extension of the knee joint, the descent speed of the simulated femur in the transition to initial contact was increased.

Because of the failure to maintain clearance between the foot and the force plate in the swing phase with the BASS, moreover, the vertical movement in the mid swing was increased to $2.5 \mathrm{~cm}$. This need apparently reflects a characteristic BASS difficulty of establishing clearance from the floor in the swing phase with single-axis knee joints. In the 3R60, the knee axis is a link mechanism, and the occurrence of simulated crus shortening in the swing phase facilitates maintenance of clearance between the foot and floor. In the BASS, in contrast, the single-axis rotation-centered structure prevents simulated crus shortening in the swing phase, which apparently results in an inability to maintain clearance between the foot and floor in the swing phase and thus led to an increased distance of vertical movement during the mid swing.

With these fine adjustments, visual observation from the sagittal plane confirmed that foot and floor clearance was maintained in initial contact, full plantar impact in the mid stance, toe off, and swing phase of each gait cycle (Fig. 4), and it was therefore concluded on the basis of this observation that simulated gait action could be accomplished. 


\section{Study for the Development of A System of Measurement of the Dynamic Characteristics of Trans-Femoral Prostheses: Measurements of Differing Knee Joint Characteristics}

\section{B. Ground Reaction Force}

Fig. 5 shows the waveform of the ground reaction force in the vertical direction, with each knee joint. With both, as shown, it was possible to achieve the bimodal form that is characteristic of reaction force in gait. The differing values of the two peaks may be an effect of motor position control resolution. In the present system, the position of the motor for simulated femoral vertical movement position can be set at intervals of $1 \mathrm{~mm}$. In the simulated gait action, however, the ground reaction force actually varied substantially with a lowering of just $1 \mathrm{~mm}$ in position depending on the state of the contact of the foot with the force plate. The ground reaction force also varies somewhat with the state of contact

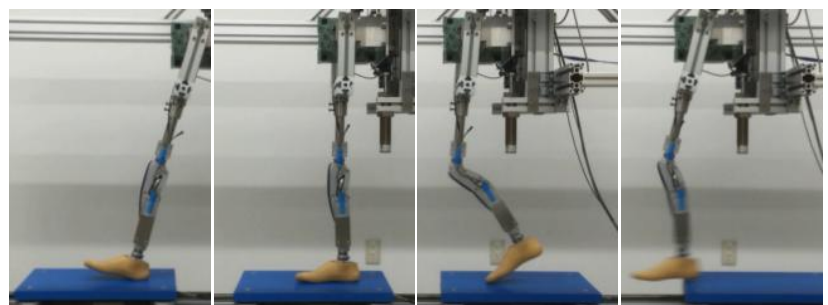

(a) Simulated gait action with 3R60

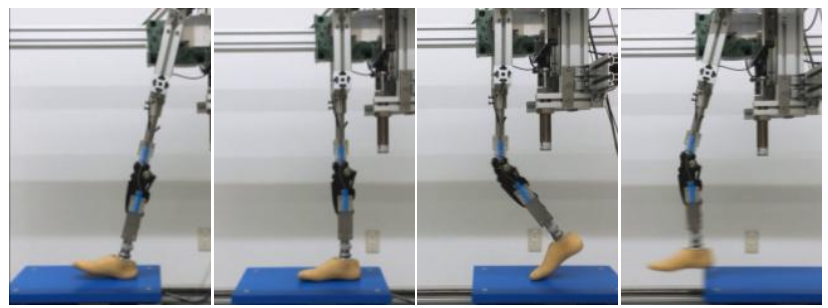

(b) Simulated gait action with BASS

Fig. 4. Simulated gait actions with different knee joints (From left: Initial contact, Mid stance, Toe off, Mid swing)

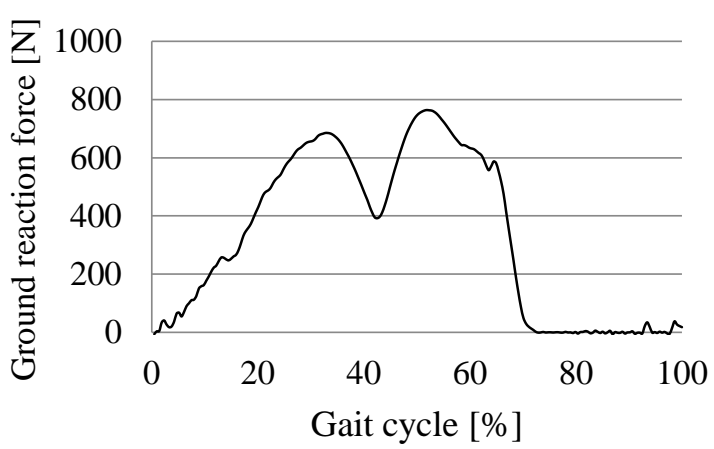

(a) Ground reaction force waveform with 3R60

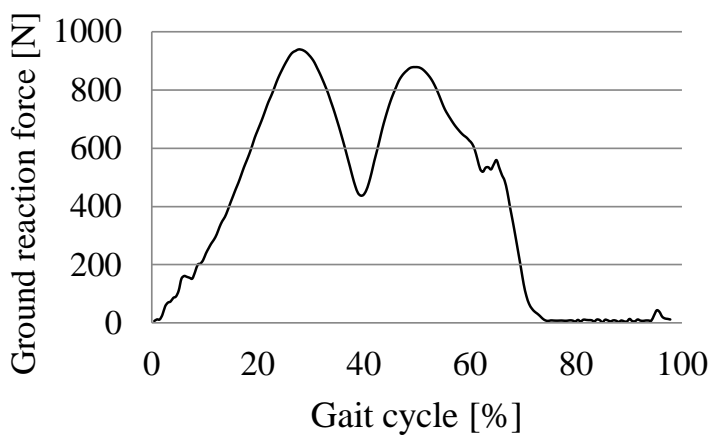

(b) Ground reaction force waveform with BASS

Fig. 5. Ground reaction force waveform with each knee between the foot and the force plate, but enablement of a finer setting of position would presumably facilitate a closer approach to the ground reaction force value. Variation is also visible immediately after initial contact, but it is attributable to slight divergence in contact between the foot and force plate and can presumably be resolved by more rigorous setting of the speed of forward movement of the force plate and the timing of foot contact.

Differences in characteristics were found on interchange between the prosthetic knee joints. A difference between the initial contact and the mid stance in ground reaction force was observed with the 3R60, but it could not be confirmed with the BASS. Its visible occurrence is apparently a reflection of the 3 R60 bouncing mechanism. The bouncing mechanism presumably reduces vertical movement of the hip joint in the initial stage of the stance phase after initial contact by lightly flexing the knee joint, and it stimulates gait with low energy consumption in able-bodied individuals by reducing the vertical movement of the center of mass. The absence of knee-joint flexing after this light flexing was presumably reflected in the ground reaction force. A variation similar to this variation in ground reaction force can be seen in gait by an amputee with a trans-femoral prostheses including the 3R60. These results indicate that the characteristics that will be exhibited by knee joints when used can effectively be measured with the present system.

\section{Knee-joint Angle}

Fig. 6 shows the variations in knee-joint angle. In the simulated gait with the prosthetic lower extremity using the 3R60, light flexing of the knee joint can be seen in the transition from initial contact to mid stance. This presumably represents the characteristic light flexing induced by the bouncing mechanism described in the earlier discussion of the ground reaction force. With the BASS, in contrast, it was found that flexion does not occur from initial contact to mid stance. This presumably reflects the knee buckling prevention function of its weight stabilizing mechanism and the resulting maintenance of full extension.

The maximum flexion angle in the BASS was approximately $10^{\circ}$ larger than in the $3 \mathrm{R} 60$. This is apparently attributable to the lightweight pneumatic control construction of the BASS, which, from the perspective of the mechanism, may be expected to result in a structure that flexes relatively easily and thus in a greater flexion angle.

\section{Relation of Hip Joint Angle to Knee Joint}

In able-bodied gait, the hip joint extends fully in the late stage of the swing phase, and knee joint flexion occurs simultaneously with the beginning of hip-joint extension following initial contact. It is known that prosthesis users show the same tendency. Examination for this relationship in simulated gait with the present system showed a similar tendency (Fig. 7). This accordingly represents one more aspect in which simulated gait action in the present system can closely approach that of actual gait. 
Taken together, the results show that the simulated gait action performed in the present system closely matches the

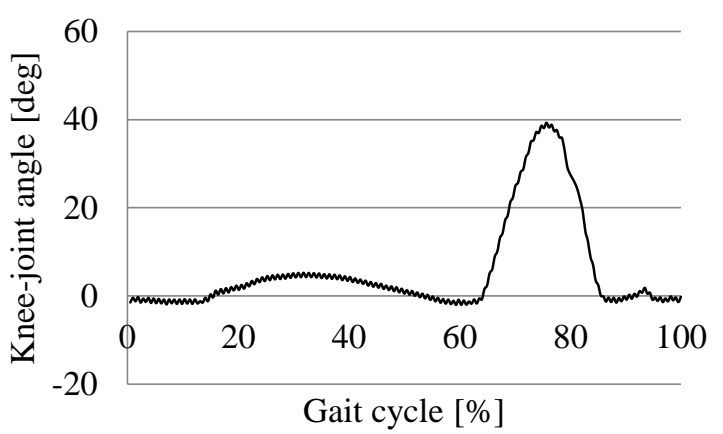

(a) Knee-joint angles with 3R60

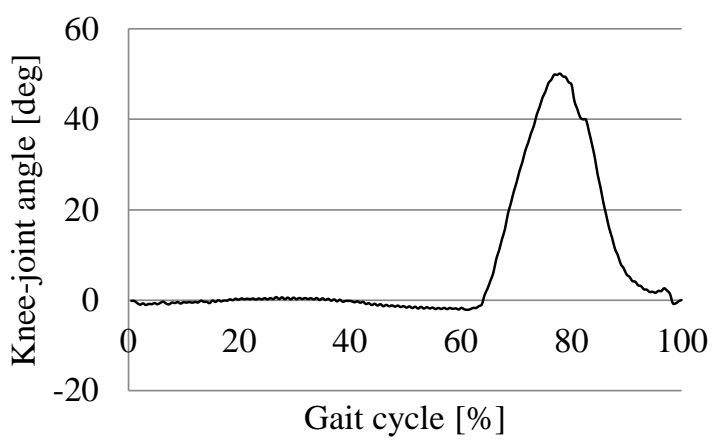

(b) Knee-joint angles with BASS

Fig. 6. Knee-joint angles with each knee joint

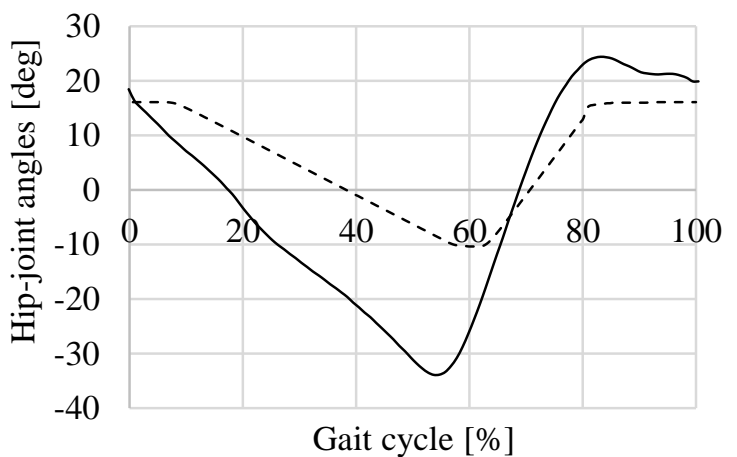

(a) Hip-joint angles (Solid curve: Femur prosthesis user; Dashed curve: Present system (both 3R60 and BASS))

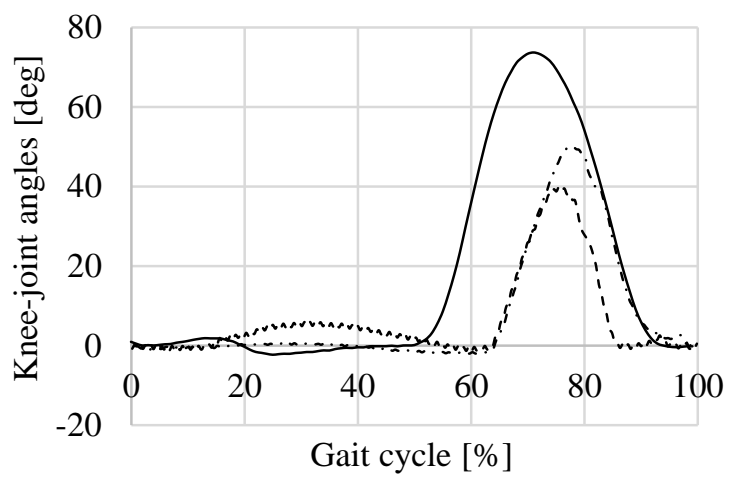

(b) Knee-joint angles

(Solid curve: Femoral prosthesis user;

Dashed curve: 3R60; Chain curve: BASS)

Fig. 7. Hip-joint and knee-joint angles in the gait cycle actual gait action, and it enables measurements yielding data that effectively reflect knee-joint characteristics.

\section{SUMMARY}

The present study investigated the validity of findings on gait actions with prosthetic knee joints of different types in a system that accommodates simulated gait with trans-femoral prostheses, with the results showing tendencies similar to those of actual prosthetic users in ground reaction force, timing of knee-joint flexion and extension, and other aspects, and it was confirmed that simulated gait can be effectively performed in the present system and the characteristics of knee joints effectively measured.

Because of the early stage of the experiments in the present study, they were performed under limited conditions in hip-joint flexion and extension angles and cadence, and it remains necessary to verify the simulated gait actions under conditions closer to those of actual human gait. For system improvement and high reliability, further investigations on gait action testing will also be necessary with trans-femoral prostheses including knee joints differing in a greater range of characteristics.

\section{REFERENCES}

[1] S. Sekikawa and S. Morimoto, "Measurement and evaluation of stance phase control function of external prosthetic knee joints," Society of Biomechanisms Japan, 14, 1998, pp. 205-216.

[2] T. Nosaka, A. Kuriyama and N. Yamasaki, "Current State of Prosthetic Knee Joints," The Japanese Association of Rehabilitation Medicine, 37, 2001, pp. 460-464.

[3] H. Yoshida, S. Morimoto and Y. Hashimoto, "Development of a Loading Test Device for Prosthetic Feet Based on ISO22675 (Dynamic Roll-over Analysis Using 3-Axis Pylon Load Cell)." Transactions of the Japan Society of Mechanical Engineers, Series C, Vol.78, No.795, 2012, pp. 3642-3649.

[4] J. Rose, J. G. Gamble, Human Walking (Third Edition), Tokyo: Ishiyaku Publishers,Inc., 2009.

[5] T. Tsuruga, A. Takashima, K. Yoshida, H. Matsubara, T. Kobayashi, K. Kon, S, Shimizu and T. Nosaka, "Characteristic inspection of the movable force plate in the trans-femoral prostheses evaluation system." The Clinical Gait Analysis Forum of Japan, 2(2), 2015, pp. 9-16.

[6] Otto Bock, Product Information, ottobock, 2014-2015, pp. 101-102.

[7] Imasen Electric Industrial Co., Ltd., LAPOC SYSTEM CATALOG, Imasen Electric Industrial Co., Ltd., 2013, pp. 25-35.

[8] T. Tsuruga, H. Iino, A. Takashima, K. Yoshida, H. Matsubara, T. Kobayashi, K. Kon, S, Shimizu and T. Nosaka, "Development of the dynamic characteristics measurement system of trans-femora prostheses. - Examination and adjustment of control parameter -.' The Clinical Gait Analysis Forum of Japan, 4(2), 2017, pp. 13-21.

Takeshi Tsuruga is an associate professor at The Hokkaido University of Science. He received his $\mathrm{PhD}$ from Hokkaido University, in 2001. His research of interest includes Assistive Engineering, Ergonomics and Walking Aid in Snowy Cold Region.

Hiroyuki Iino received his Master's Degree from The Hokkaido University of Science in 2015. He is studying Prosthetics and Orthotics, Assistive Engineering and Ergonomics at the Imasen Engineering Corporation.

Akihiko Takashima is an assistant professor at The Hokkaido University of Science. In 2004 he received a doctorate from Muroran Institute of Technology. His research of interest includes Robotics, especially Motion Control of Nonholonomic Robots. 